\title{
Rapid In Situ Melting and Revitrification as an Approach to Microsecond Time- Resolved Cryo-Electron Microscopy
}

\author{
Jonathan M. Voss, Oliver F. Harder, Pavel K. Olshin, Marcel Drabbels, and Ulrich J. Lorenz
}

Laboratory of Molecular Nanodynamics, École Polytechnique Fédérale de Lausanne, 1015 Lausanne, Switzerland.

While cryo-electron microscopy (cryo-EM) is rapidly becoming the dominant method in structural biology, its time resolution is currently insufficient to directly observe proteins in action, leaving our understanding of proteins fundamentally incomplete. So-called time-resolved cryo-EM achieves a timeresolution of a few milliseconds, which is notably too slow to study the dynamics of many proteins whose relevant motions typically occur on the microsecond timescale. Here, we demonstrate a novel approach to time-resolved cryo-EM that affords microsecond time resolution [1]. As illustrated in Fig. 1, our method involves melting a cryo sample in situ with a laser beam (a), which allows dynamics of the embedded particles to occur in liquid once a suitable stimulus is provided (b), for example by releasing a caged compound. While the dynamics occur, the heating laser is switched off at a well-defined point in time, causing the sample to rapidly recool (c), so that it vitrifies and the particles are trapped in their transient configurations (d), in which they can subsequently be imaged (e).

It may appear doubtful whether such a scheme could work. Usually, it is strictly avoided to warm up cryo samples, as this induces devitrification. Moreover, in order to revitrify the sample, it would be necessary to cool it very rapidly, at rates of $10^{6} \mathrm{~K} / \mathrm{s}$ or higher. We demonstrate that these obstacles can be overcome and that cryo samples can indeed be melted for durations of tens of microseconds or longer and successfully revitrified thereafter. This is possible because of the high heating and cooling rates of about $10^{8} \mathrm{~K} / \mathrm{s}$ that we achieve in our experiments, as determined from stroboscopic time-resolved experiments as well as heat transfer simulations. Crucially, the cooling time dictates how rapidly particles can be trapped in a transient configuration and therefore determines the time resolution of the experiment, which we show to be better than $5 \mu$ s.

Figure 2 shows a proof-of-principle experiment, demonstrating that our method allows particle dynamics to occur in liquid phase during the short time window created by laser melting and that the particles can subsequently be trapped in transient states during rapid revitrification. We make use of the well-known fact that proteins incur electron beam damage during cryo imaging. It is commonly believed that the vitreous ice matrix counteracts this damage and preserves the protein structure by fixing fragments in place. However, melting the sample should allow the particles to unravel as they find themselves in a liquid environment. This is exactly what we observe in our experiment. When we image a cryo sample of apoferritin (a) before melting and revitrifying it with a $15 \mu$ s laser pulse in situ (b), we find that particles in the areas previously exposed to the electron beam have disassembled and have been trapped in partially unraveled states (d,e). At the same time, particles that have not incurred beam damage are left intact (c). In conclusion, we present a novel approach for time-resolved cryo-EM that affords a time-resolution of just a few microseconds, three orders of magnitude faster than what has previously been possible. Our method provides an alternative access to in situ liquid phase experiments on short timescales. In the future, we envision to trigger a range of naturally occurring protein dynamics with biomimetic stimuli by releasing a caged compound, such as ATP, calcium ions, or peptides. 


\section{References:}

[1] JM Voss et al, ArXiv:2103.12589.

[2] This work was supported by the ERC Starting Grant 759145 and by the Swiss National Science Foundation Grant PP00P2_163681.

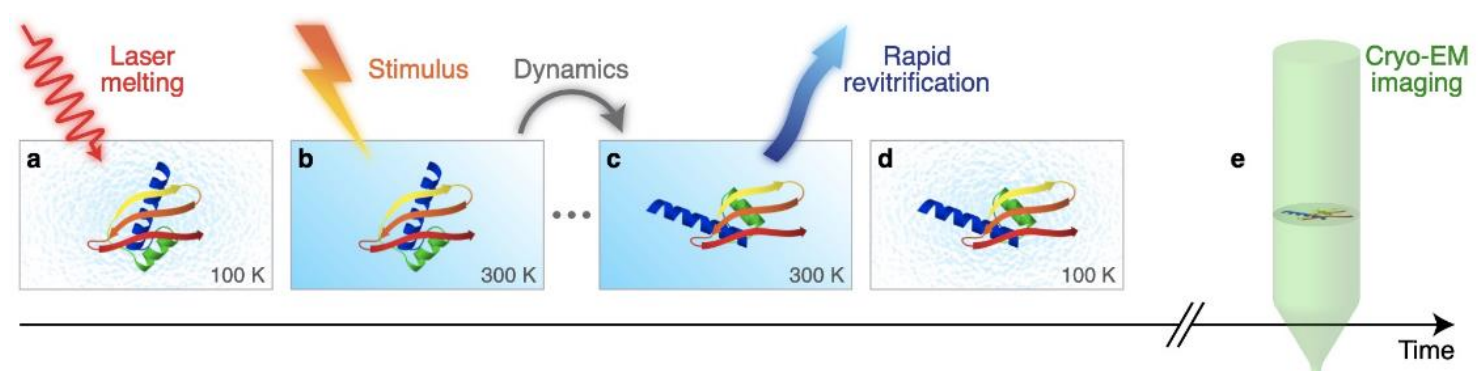

Figure 1. Experimental concept for microsecond time-resolved cryo-EM. (a) A cryo sample is melted in situ with a heating laser. (b) Once the sample is liquid and has reached room temperature, dynamics of the embedded particles are induced with an external stimulus. (c) As the particle undergoes conformational changes, the heating laser is switched off, so that the sample rapidly cools and revitrifies. (d) The particle is trapped in its transient configuration and can be subsequently imaged with conventional cryo-EM techniques (e).
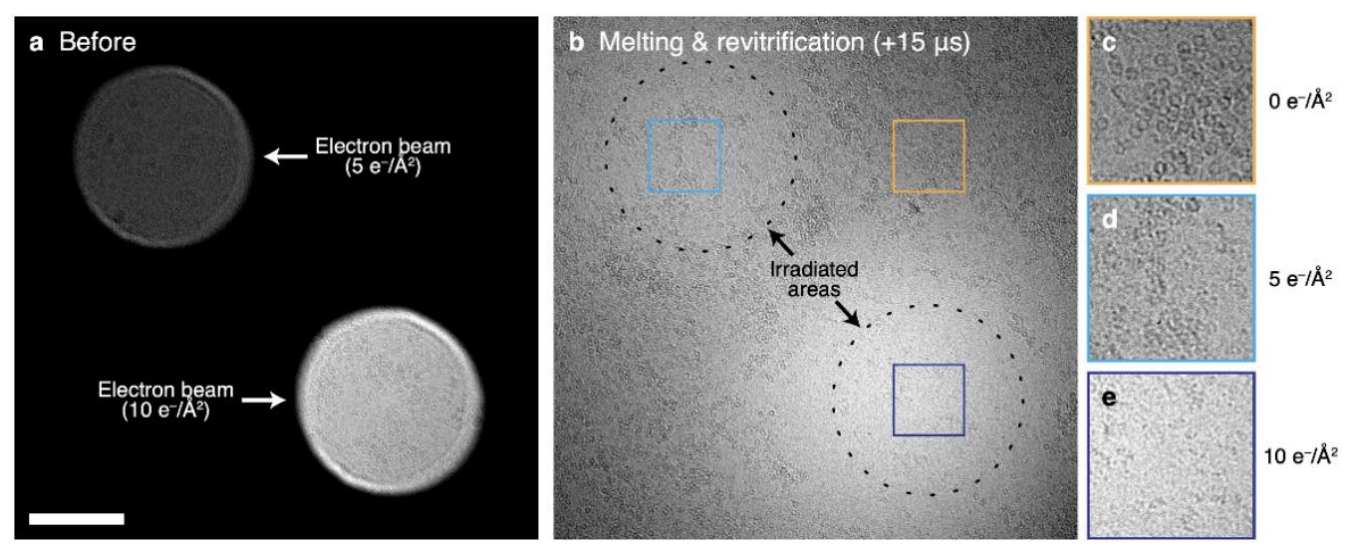

Figure 2. Proof-of-principle demonstration of microsecond time-resolved cryo-EM. (a) Micrograph of a cryo sample of apoferritin on a holey gold film. Only the top left and bottom right areas are exposed with a dose of 5 and 10 electrons/Å2, respectively. Scale bar, $200 \mathrm{~nm}$. (b) The sample is melted in situ with a $15 \mu \mathrm{s}$ laser pulse and revitrifies. (c) The particles not illuminated with the electron beam prior to melting and revitrification remain intact. In contrast, those previously damaged with the electron beam have unraveled in liquid and upon revitrification, have been trapped in partially disassembled configurations $(\mathrm{d}, \mathrm{e})$. 